\title{
Effects of dynamic myofascial release on trunk mobility and standing balance in persons with chronic nonspecific low back pain
}

\author{
Dong-Woo Lee ${ }^{a}$, Hwa-Kyung Shin ${ }^{b}$, Kwang-Su Kim ${ }^{a}$ \\ aDepartment of Physical Therapy, Graduate School, Daegu Catholic University, Gyeongsan, Republic of Korea \\ ${ }^{\mathrm{b}}$ Department of Physical Therapy, Graduate School, College of Bio and Medical Science, Daegu Catholic University, Gyeongsan, Republic of Korea
}

\begin{abstract}
Objective: Myofascial release (MFR) is used to restore tissue extensibility of the fascia tissue and is considered to be useful in a number of clinical settings, such as low back pain (LBP). Dynamic myofascial release (DMFR) is the manual therapy, which combined the conventional MFR with the joint mobilization. The purpose of this study was to investigate the effects of the DMFR on trunk mobility, and furthermore, whether the increase of trunk mobility can carry over the improvement of dynamic standing balance in persons with chronic nonspecific LBP.
\end{abstract}

Design: Randomized controlled trial.

Methods: Thirty persons with chronic non-specific LBP participated in the study and were randomly assigned to the DMFR group $(n=15)$ or the control group $(n=15)$. DMFR was performed for two sessions ( 15 minutes/session) per week for four weeks for the treatment group. Both the DMFR and control groups were allowed to perform low-intensity physical activities during the treatment period. The Modified-modified Schöber test (MMST) for trunk mobility and the Functional Reach Test (FRT) for dynamic standing balance were measured before and after the treatment period in both the DMFR group and the control group.

Results: The MMST value of DMFR group increased significantly in all trunk range of motion (flexion, extension, lateral flexion, and rotation) after treatment, compared with the control group $(p<0.05)$. Additionally, the FRT value of the DMFR group improved significantly after treatment, compared with the control group $(p<0.05)$.

Conclusions: We suggest that DMFR have a positive effect on trunk mobility and standing balance in persons with chronic LBP.

Key Words: Low back pain, Postural balance, Range of motion

\section{Introduction}

Chronic low back pain (LBP) is a significant medical problem that is common in modern society [1]. Furthermore, LBP is the second most common reason for clinical visits and the fifth most common reason for hospital visits for surgical procedures [2]. Non-specific LBP especially does not have a known pathophysiological cause, and the treatment focuses on reducing pain and increasing functional trunk mobility. Joint mobilization, myofascial release (MFR), and simple message have been applied to decrease the symptoms of LBP in clinical practice $[3,4]$.

Fascial tissue is considered to be a source of nociceptive pain (myofascial pain) in several musculoskeletal disorders including plantar fasciitis, Dupuytren's contracture, and non-specific LBP. Many therapies have been used for myofascial pain, such as varying forms of MFR, which have been based upon Rolf's structural integration model and has been developed over the past 30 years [5]. MFR involves manual application of low amplitude, long duration stretch-

Received: 1 May, 2019 Revised: 10 June, 2019 Accepted: 11 June, 2019

Corresponding author: Hwa-Kyung Shin (ORCID https://orcid.org/0000-0003-3876-0710)

Department of Physical Therapy, Graduate School, College of Bio and Medical Science, Daegu Catholic University, 13-13 Hayang-ro, Geumnak-ri, Hayang-eup Gyeongsan 38430, Korea

Tel: 82-53-850-2532 Fax: 82-53-359-6772 E-mail: hkshin1@cu.ac.kr

(c) This is an Open-Access article distributed under the terms of the Creative Commons Attribution Non-Commercial License (http://creativecommons.org/licenses/ by-nc/4.0) which permits unrestricted non-commercial use, distribution, and reproduction in any medium, provided the original work is properly cited.

Copyright () 2019 Korean Academy of Physical Therapy Rehabilitation Science 
es to the fascial muscles in static posture [6].

Although there has been much research on the therapeutic effect of the MFR, there is a lack of research data on dynamic techniques, which is MFR combined with joint mobilization. Dynamic MFR (DMFR) is similar to joint mobilization for ROM increase and with the MFR for fascia release. However, MFR is a passive approach by therapists in the static posture, while DMFR differs in that it is a dynamic approach in which the patient is actively involved. Therefore, DMFR may be more effective in terms of stimulating proprioception and creating non-local systemic effects [7].

The purpose of this study was to investigate the effects of DMFR on trunk mobility, and furthermore, whether the increase of trunk mobility can carry over in improving dynamic stance balance. It is anticipated that there will be an increase in thoracolumbar ROM and distance of functional reach after DMFR.

\section{Methods}

\section{Participants}

Thirty persons with chronic nonspecific LBP were recruited and were randomly assigned to either the DMFR group $(n=15)$ or the control group $(n=15)$ (Table 1$)$. Inclusion criteria were the following: (1) $>12$ weeks from LBP onset; (2) Visual Analog Scale scores of less than or equal to 5 and pain that does not aggravate the sitting position since the patients are required to perform DMFR; (3) no severe complications; (4) patients with no arterial hypertension and with progressive neurological deficits; (5) those who are not under pharmacological or psychiatric treatment. Written and verbal information was given to all participants and informed consent forms were signed. This study was approved by the institutional ethical committee of the Daegu Catholic University (IRB No. CUIRB-2018-0036).

Table 1. General characteristics of participants

$(\mathrm{N}=30)$

\begin{tabular}{lcc}
\hline \multicolumn{1}{c}{ Variable } & DMFR $(\mathrm{n}=15)$ & Control $(\mathrm{n}=15)$ \\
\hline Sex (male/female) & $7 / 8$ & $7 / 8$ \\
Age $(\mathrm{y})$ & $61.01(7.86)$ & $62.05(5.79)$ \\
Height $(\mathrm{cm})$ & $163.81(6.85)$ & $164.39(5.34)$ \\
Weight $(\mathrm{kg})$ & $64.34(4.69)$ & $65.26(3.26)$ \\
\hline
\end{tabular}

Values are presented as number only or mean (SD). DMFR: dynamic myofascial release.

\section{Study design}

This study included an experimental design conducted was a triple-blind (participants, researcher, and therapist), randomized, and controlled design. Both the DMFR and control group were allowed to perform low-intensity physical activities and routine lumbar stabilization exercises during the treatment phase. DMFR was performed over two sessions (15 minutes/session) per week for four weeks. A professional physiotherapist with a clinical experience of over five years performed the DMFR. After the treatment period, trunk range of motion (ROM) (flexion, extension, lateral flexion, and rotation) and the Functional Reach Test (FRT) was performed to measure dynamic standing balance.

\section{Dynamic myofascial release}

DMFR involves a manual application of low amplitude, long duration stretch to the fascia and muscle, between the levels T6-12. Participants were instructed to relax as much as possible, and the therapist proceeded to smoothly move the joints in a diagonal or horizontal direction at a slow rate within the ROM. The therapist repeatedly pushed, pulled, or shook the joint area about three to five times for about $3 \mathrm{sec}-$ onds with slight motion at the end of ROM (Figure 1). In step 1 , the therapist stood facing the participants and supported one arm of the participants to move. The therapist pulled and pushed gently on the side of the shoulder. When the participant shifted the weight forward, the therapist simultaneously moved to the front side slowly with breathing (Figure 1A). In step 2, the participant put both hands on his neck, and the therapist flexed the knee to $90^{\circ}$ and placed the participant's elbows on his lap. When the participant shifted his body weight forward, the therapist slowly adjusted the patient's breathing (Figure 1B). In step 3, the therapist supported the patient's upper flexed knee on his thigh. He made movements of the pelvis and lower limb which was similar to the gait pattern (Figure 1C). In step 4, the therapist supported the patient' upper flexed knee using one hand. Therapist pushed and pulled the iliac crest to the anterior, posterior, upward, and downward directions (Figure 1D). Finally, the patient was prone-lying the therapist fixed one of the shoulder or pelvis and then moved the other part for counter rotation between the shoulder and the pelvis (Figure 1E).

\section{Modified-modified Schöber test}

Trunk mobility was measured with the Modified-modi- 


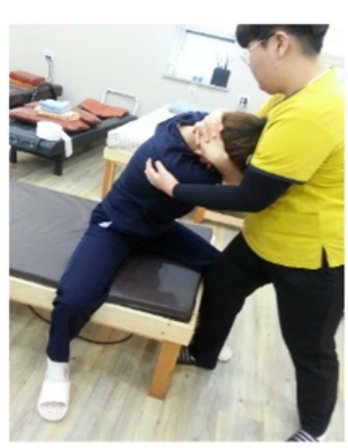

A

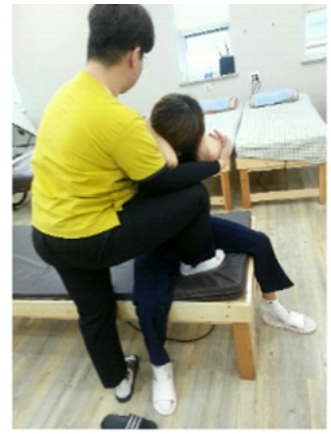

B

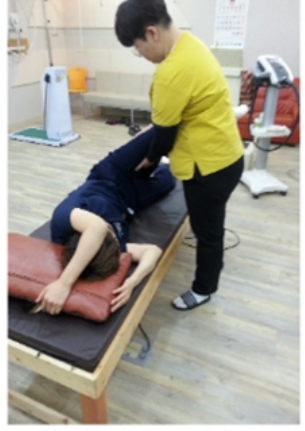

C

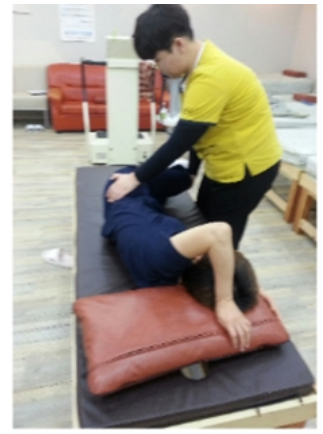

D

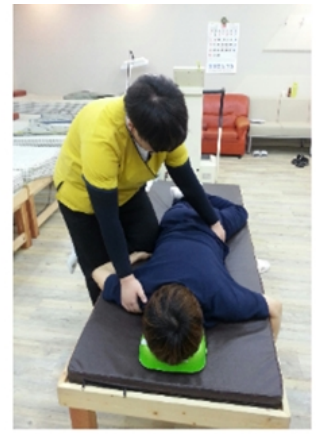

E

Figure 1. Dynamic myofascial release. (A) Step 1, (B) step 2, (C) step 3, (D) step 4, (E) step 5.

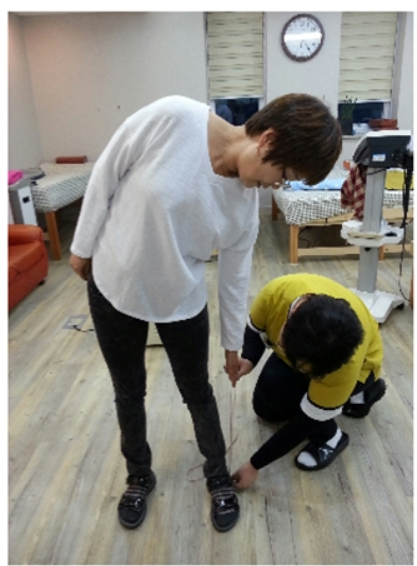

A

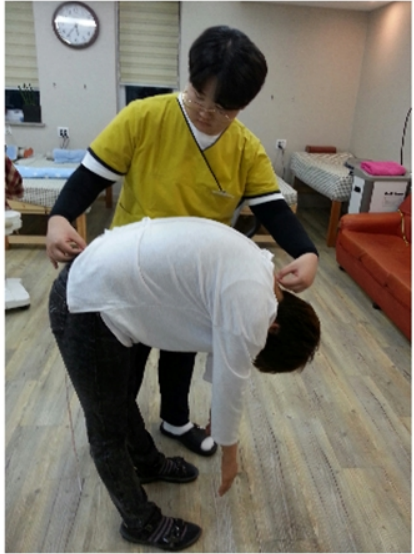

B

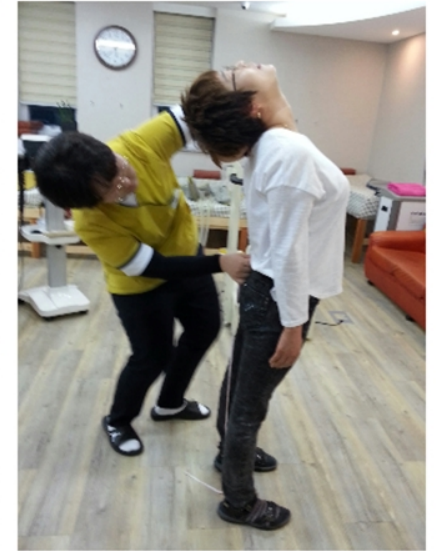

C

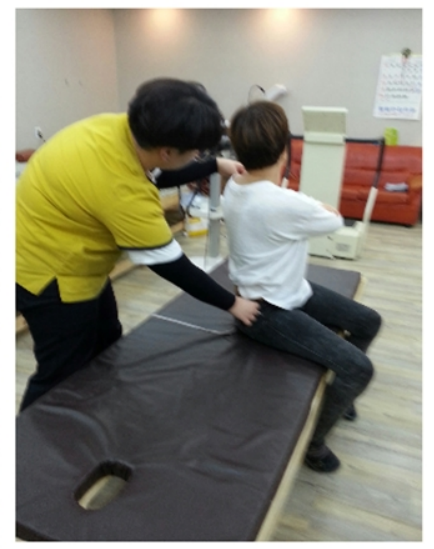

D

Figure 2. Modified-modified Schöber test. (A) Lateral flexion, (B) flexion, (C) extension, (D) rotation.

fied Schöber test (MMST) using tape measurement (Figure 2). The representative value was defined as the mean value of three measurements [8]. For lateral flexion of the trunk, the participant stood with their feet shoulder-width apart (Figure 2A). The examiner measured the changes in the level of the tip of the middle finger on the thigh after maximal lateral flexion. For flexion of the trunk, the participant stood with placing both hands on the iliac crests and extending the knee (Figure 2B). The examiner recorded the changes in the distance between the spinous processes of $\mathrm{C} 7$ and $\mathrm{S} 2$ after maximal flexion. As the same way, trunk extension was measured (Figure 2C). For rotation of trunk, the participant was sitting with the feet supported on a stool with arms crossed in front of the chest (Figure 2D). The participant held the end of the tape on the lateral aspect of the acromion process, and the examiner held onto the other end of the tape on the greater trochanter. The examiner measured the changes in the distance between the acromion process and greater trochanter after maximal trunk rotation (Figure 2E). The MMST demonstrated moderate validity $(\mathrm{r}=0.67 ; 95 \%$ confidence interval [CI], 0.44-0.84), excellent reliability (intra: intra-class correlation coefficient $[\mathrm{ICC}]=0.95 ; 95 \% \mathrm{CI}, 0.89-0.97$; inter: $\mathrm{ICC}=0.91 ; 95 \% \mathrm{CI}, 0.83-0.96$ ) and a MMDC of $1 \mathrm{~cm}$ $[9,10]$.

\section{Functional Reach Test}

Dynamic standing balance was measured with the FRT. Participants were instructed to reach forward with the left arm horizontally while maintaining a fixed base of support to evaluate standing balance $[11,12]$. A $150 \mathrm{~cm}$ yardstick was mounted horizontally on the wall at the height of the acromion process. Reaching distance was measured as the displacement of the finger between the starting and end position. FRT demonstrated moderate validity and high reliability [13]. 
Table 2. Comparison of difference values of pre and post-test between the DMFR group and control group on MMST and FRT

\begin{tabular}{|c|c|c|c|c|c|c|c|}
\hline \multirow{2}{*}{ Variable } & \multicolumn{3}{|c|}{ DMFR $(n=15)$} & \multicolumn{3}{|c|}{ Control $(n=15)$} & \multirow{2}{*}{$\mathrm{t}(p)$} \\
\hline & Pre-test & Post-test & Change & Pre-test & Post-test & Change & \\
\hline \multicolumn{8}{|l|}{ MMST $(\mathrm{cm})$} \\
\hline Flexion & $4.05(0.55)$ & $8.53(1.25)$ & $4.48(1.10)$ & $5.29(1.06)$ & $5.83(1.04)$ & $0.55(0.22)$ & $13.36(<0.001)$ \\
\hline Extension & $5.29(1.06)$ & $13.80(1.15)$ & $1.18(0.42)$ & $9.34(0.96)$ & $10.06(1.08)$ & $0.46(0.19)$ & $6.06(<0.001)$ \\
\hline Lateral flexion & $8.60(0.78)$ & $13.80(1.15)$ & $5.20(0.68)$ & $9.34(0.96)$ & $10.06(1.08)$ & $0.72(0.30)$ & $22.51(<0.001)$ \\
\hline Rotation & $2.03(0.40)$ & $3.15(0.45)$ & $1.13(0.18)$ & $2.25(0.24)$ & $2.71(0.28)$ & $0.46(0.26)$ & $7.92(<0.001)$ \\
\hline FRT (cm) & $15.60(2.99)$ & $19.66(2.61)$ & $4.06(0.90)$ & $15.59(2.98)$ & $16.34(2.90)$ & $0.75(0.21)$ & $13.36(<0.001)$ \\
\hline
\end{tabular}

Values are presented as mean (SD).

DMFR: dynamic myofascial release, MMST: Modified-modified Schöber test, FRT: Functional Reach Test.

\section{Statistical analysis}

The Shapiro-Wilk test was used to confirm that the data was normally distributed ( $p>0.05$ ), thus justifying the use of parametric tests. The independent t-test was used to compare the general characteristics between the DMFR group and the control group and compare the difference value of pre and post-test between groups on the FRT and trunk ROM. The IBM SPSS Statistics ver. 21.0 software (IBM Co., Armonk, NY, USA) was used for statistical analysis and a CI of $95 \%$ ( $p$-value $<0.05)$ was used.

\section{Results}

There were no significant differences in the general characteristics of the participants between the DMFR group and the control group ( $p>0.05$, Table 1$)$. The DMFR group increased significantly, compared with the control group in all trunk ROM; flexion, extension, lateral flexion, and rotation $(p<0.05)$. Additionally, the DMFR group increased significantly, compared with the control group on the FRT results $(p<0.05$, Table 2$)$.

\section{Discussion}

In this study, trunk ROM of the DMFR group increased significantly after the intervention. We found that the DMFR, which was a combination of MFR and joint mobilization, was useful for increasing trunk ROM. DMFR was applied with focus on the thoracic spine than the painful lumbar spine itself. DMFR was first applied to the thoracic spine and then to the lumbar spine. LBP patients have to correct abnormal trunk movement pattern, which can be improved by changing trunk muscle activities or muscle lengths. Therefore, the therapist should seek how to apply substituted thoracic trunk movements when lumbar spine movement is limited in persons with LBP $[14,15]$. It was reported that joint mobilization applied to the thoracic region could help to maintain proprioception and control pain in the lumbar spine in persons with LBP [16]. Some studies reported that joint mobilization on the thoracic spine was more effective than direct motion at the lumbar spine on improving lumbar stability [17].

Our study showed that trunk mobility increased significantly in the DMFR group compared with the control group and that dynamic standing balance improved significantly in the DMFR group compared to the control group. DMFR applied to the local area promoted an increase in thoracolumbar ROM by the biomechanical effect as well as improved dynamic standing balance by system effect $[6,18]$. That is, when stimulated, the Ruffini corpuscles (mechanoreceptors) have been associated with a further decrease in activity of the sympathetic nervous system of the automomic nervous system, as fascia has a high density of free nerve endings that belong to the sympathetic nervous system, leading to a more relaxed parasympathetic response, thus allowing for greater ROM and better dynamic stance balance [5].

Some studies reported that elderly patients with non-specific LBP decreased their reliance on ankle strategy and hip strategy proprioceptive signals during balance control [19]. Some studies have shown that MFR or joint mobilization is effective in improving the balance of persons with non-specific LBP [20]. Also, the myofascial connections (myofascial trains or sequences) could be directly effective in the organization of movement and muscular force transmission, and 
finally the released stiffness could have an influence on motor control and improve balance [21]. Similarly, our current study showed increased trunk motility could lead to improvements in functional dynamic standing balance.

There were some limitations to this study. Firstly, we did not distinguish the various pathological causes of LBP. Secondly, there was a small sample of only 15 participants for each group, warranting further studies with a full sample size calculated by a power analysis to follow after this study to ensure the generalization of these results.

\section{Acknowledgements}

This work was supported by research grants from the Daegu Catholic University in 2018.

\section{Conflict of Interest}

The authors declared no potential conflicts of interest with respect to the authorship and/or publication of this article.

\section{References}

1. Ha SY, Han JH, Sung YH. Effects of ankle strengthening exercise program on an unstable supporting surface on proprioception and balance in adults with functional ankle instability. $\mathrm{J}$ Exerc Rehabil 2018;14:301-5.

2. Hemming R, Sheeran L, van Deursen R, Sparkes V. Non-specific chronic low back pain: differences in spinal kinematics in subgroups during functional tasks. Eur Spine J 2018;27:163-70.

3. Stanek J, Sullivan T, Davis S. Comparison of compressive myofascial release and the Graston Technique for improving ankle-dorsiflexion range of motion. J Athl Train 2018;53:160-7.

4. Climent JM, Kuan TS, Fenollosa P, Martin-Del-Rosario F. Botulinum toxin for the treatment of myofascial pain syndromes involving the neck and back: a review from a clinical perspective. Evid Based Complement Alternat Med 2013. doi: 10. 1155/2013/381459. [Epub ahead of print]

5. Cathcart E, McSweeney T, Johnston R, Young H, Edwards DJ. Immediate biomechanical, systemic, and interoceptive effects of myofascial release on the thoracic spine: a randomised controlled trial. J Bodyw Mov Ther 2019;23:74-81.

6. Rajasekar S, Marchand AM. Fascial Manipulation for persistent knee pain following ACL and meniscus repair. J Bodyw Mov Ther 2017;21:452-8.
7. MacDonald N, Baker R, Cheatham SW. The effects of instrument assisted soft tissue mobilization on lower extremity muscle performance: a randomized controlled trial. Int J Sports Phys Ther 2016;11:1040-7.

8. Clarkson HM. Musculoskeletal assessment: joint motion and muscle testing. 3rd ed. Philadelphia (PA): Wolters Kluwer Lippincot Williams \& Wilkins; 2013.

9. Tousignant M, Poulin L, Marchand S, Viau A, Place C. The Modified-Modified Schober Test for range of motion assessment of lumbar flexion in patients with low back pain: a study of criterion validity, intra- and inter-rater reliability and minimum metrically detectable change. Disabil Rehabil 2005;27:553-9.

10. Williams R, Binkley J, Bloch R, Goldsmith CH, Minuk T. Reliability of the modified-modified Schöber and double inclinometer methods for measuring lumbar flexion and extension. Phys Ther 1993;73:33-44.

11. Khalifeloo M, Naghdi S, Ansari NN, Akbari M, Jalaie S, Jannat D, et al. A study on the immediate effects of plantar vibration on balance dysfunction in patients with stroke. J Exerc Rehabil 2018;14:259-66.

12. Rosa MV, Perracini MR, Ricci NA. Usefulness, assessment and normative data of the Functional Reach Test in older adults: a systematic review and meta-analysis. Arch Gerontol Geriatr 2019;81:149-70.

13. Katz-Leurer M, Fisher I, Neeb M, Schwartz I, Carmeli E. Reliability and validity of the modified functional reach test at the sub-acute stage post-stroke. Disabil Rehabil 2009;31:243-8.

14. Taniguchi M, Tateuchi H, Ibuki S, Ichihashi N. Relative mobility of the pelvis and spine during trunk axial rotation in chronic low back pain patients: a case-control study. PLoS One 2017;12: e0186369.

15. Yoo WG. Effect of thoracic movement-mediated training on back pain and trunk range of motion in a patient with lower back pain. J Phys Ther Sci 2015;27:2665-6.

16. LeBauer A, Brtalik R, Stowe K. The effect of myofascial release (MFR) on an adult with idiopathic scoliosis. J Bodyw Mov Ther 2008;12:356-63.

17. Sung YB, Lee JH, Park YH. Effects of thoracic mobilization and manipulation on function and mental state in chronic lower back pain. J Phys Ther Sci 2014;26:1711-4.

18. Schleip R. Fascial plasticity ${ }^{-}$a new neurobiological explanation: part 1. J Bodyw Mov Ther 2003;7:11-9.

19. Ito T, Sakai Y, Morita Y, Yamazaki K, Igarashi K, Nishio R, et al. Proprioceptive weighting ratio for balance control in static standing is reduced in elderly patients with non-specific low back pain. Spine (Phila Pa 1976) 2018;43:1704-9.

20. Park DJ, Hwang YI. A pilot study of balance performance benefit of myofascial release, with a tennis ball, in chronic stroke patients. J Bodyw Mov Ther 2016;20:98-103.

21. Myers TW. Anatomy trains: myofascial meridians for manual and movement therapists. 3rd ed. Edinburgh: Churchill Livingstone/Elsevier; 2014. 